\title{
Multi-method confirmatory factor analyses of puberty in early adolescent girls
}

\author{
Running title: Puberty CFA in Early Adolescent Girls
}

\begin{abstract}
Authors: Michelle L. Byrne ${ }^{1}$, Samantha J. Chavez ${ }^{1}$, Nandita Vijayakumar ${ }^{2}$, Theresa W. Cheng ${ }^{1}$, John C.
\end{abstract} Flournoy $^{3}$, Marjolein E. A. Barendse ${ }^{1}$, Elizabeth A. Shirtcliff ${ }^{4}$, Nicholas B. Allen ${ }^{1}$, Jennifer H. Pfeifer ${ }^{1}$

1. Department of Psychology, The University of Oregon, Eugene, OR

2. School of Psychology, Deakin University, Victoria, Australia

3. Department of Psychology, Harvard University, Cambridge, Massachusetts

4. Human Development and Family Studies, lowa State University, Ames, lowa

Abbreviations: DHEA = dehydroepiandrosterone; $\mathrm{T}$ = testosterone; $\mathrm{E} 2$ = estradiol; PDS = pubertal development scale; LD $=$ Morris \& Udry Line Drawings; $A D R=$ adrenarche factor; GON = gonadarche factor; PUB = puberty factor

\section{Abstract (full)}

Correctly measuring and operationalizing pubertal processes during development is important for capturing variation in both normative development and trajectories associated with adverse outcomes. The aim of the current study was to understand how this variance may be captured by different pubertal processes (adrenarche and gonadarche) and how multiple methods may contribute to variance (and how they are correlated). The study explored multi-method cross-sectional pubertal data, including self-reported physical characteristics from the Pubertal Development Scale (PDS) and Morris \& Udry line drawings, and levels of hormones dehydroepiandrosterone (DHEA), testosterone (T), and estradiol (E2) in saliva and hair, from a cohort of 174 early adolescent girls aged 10-13 years. Authors developed a theory-driven structural equation modelling framework of puberty for girls that includes multiple measures of puberty and considers both processes of adrenarche and gonadarche. Self-reported puberty and hormone levels from saliva and hair can estimate an overall pubertal process as well as each process of adrenarche and gonadarche. The self-reported PDS item on height growth had the lowest standardized loading of the self-report items. In full models with all measures, the more parsimonious one-factor model was not a significantly worse fit than the two-factor model. The shared variance between the hormonal latent puberty factor and the self-reported latent puberty factor was $32 \%$. This suggests fairly good reliability between hormones and self-report puberty data but also suggests that measuring puberty via hormones and via self-report questionnaires is not entirely redundant, and models of puberty require collecting both. Exploratory analyses showed $34 \%, 48 \%$, and $52 \%$ shared variance between chronological age and the adrenarcheal, gonadarcheal, and overall pubertal factors, respectively, suggesting that there is additional variance in these pubertal processes that is not explained by age alone. When controlling for age, the adrenarcheal and gonadarcheal factors were still strongly associated ( $90 \%$ shared variance), suggesting that these factors are correlated when calculated as pubertal timing (i.e., stage compared to same-age peers), as well. Finally, correlations with age were higher when using the latent factors, whether for self-report questionnaire data alone, or for hormones, and correlations were highest when using all available self-report and biological measures.

\section{Abstract (short)}

This structural equation modelling study explored how variance in pubertal development is captured by different processes (adrenarche and gonadarche) and methods, using cross-sectional pubertal data from a cohort of 174 early adolescent girls aged 10-13 years. Self-reported puberty and hormone levels from saliva and hair can estimate an overall pubertal process as well as each process of adrenarche and gonadarche. The self-reported PDS item on height growth had the lowest standardized loading of the self-report items.

Hormonal and self-reported latent puberty factors had $32 \%$ shared variance, suggesting that research should collect both. There was $34 \%, 48 \%$, and $52 \%$ shared variance between chronological age and the adrenarcheal, gonadarcheal, and pubertal factors, respectively, suggesting these processes are not explained by age alone. 
In developmental science, measuring maturation in adolescence is complicated. Researchers often use age as an index for maturational change, but chronological age is only a proxy for a range of experiences and stages in development (Wohlwill \& Palermo, 2014). For example, pubertal stage is a better predictor than chronological age of depression in girls (Angold et al., 1998). Puberty is a sensitive period of life for neurobiological development when major changes in social, physical, and hormonal development occur (Ladouceur et al., 2012; Paus et al., 2008). Correctly measuring and operationalizing pubertal processes during development is important for capturing variation that may be associated with adverse experiences and outcomes (Mendle et al., 2019). It is essential for puberty research to understand 1) how this variance may be captured by different pubertal processes and 2) how multiple methods may contribute to variance in development (and how they are correlated).

There are two separable phases in pubertal development: adrenarche, which is triggered by the maturation of the zona reticularis of the adrenal gland, and gonadarche, associated with the maturation of the hypothalamic-pituitary-gonadal axis. The processes of adrenarche and gonadarche are activated and controlled by separate mechanisms (Counts et al., 1987). There is evidence that later stage (where an individual is in the pubertal process at a certain time point) and early timing (status relative to peers of the same age) of gonadarche are predictive of mental health problems (e.g., (Angold et al., 1998; Oldehinkel et al., 2011). There is less work examining the effect of adrenarcheal processes on mental health, even though this period of development coincides with sensitive periods of brain development and may have evolutionary advantages for social learning during this time of transition from childhood to adolescence (Campbell, 2006, 2011). A meta-analysis of 17 studies provided initial evidence that earlier adrenarcheal timing is associated with more mental health problems, which may be mediated by neurobiological development (Byrne et al., 2017).

Researchers can measure pubertal status in a number of ways, including with pubertal hormones such as dehydroepiandrosterone (DHEA, adrenal in both sexes), testosterone (adrenal in girls), and estradiol (gonadal in girls), as well as with physical characteristics such as breast/genital development, pubic hair growth, skin changes, and body odor, either observed or self-reported. Hormones have the advantage of being objective, "biological" measures that advance pubertal maturation, but measuring hormones or physical characteristics presents various challenges. For example, measuring physical changes in research studies should be done sensitively or it can obscure or bias estimates of development in certain groups of adolescents (Deardorff et al., 2019). Additionally, we often only observe physical changes related to adrenarche, such as skeletal maturation and body odor, well after hormone levels increase (Wan, 2012). Research studies that only collect data on physical characteristics of puberty may therefore not have precise temporal information about this process. Hormones add to an important multi-method assessment of puberty.

However, there are considerable methodological challenges with measuring hormones. Levels fluctuate depending on other factors that are not necessarily inherent to pubertal processes, such as acute stress (Lennartsson et al., 2012), nutrition (Soliman et al., 2014), and exercise (Hackney \& Lane, 2015). Hormone levels can be measured in a wide range of biological specimens, commonly including blood, saliva, urine, and hair. Each of these biospecimens has advantages depending on the specific research question (repeated measures, cumulative exposure, invasiveness for certain groups of participants). Blood and saliva samples are the most commonly used in research studies of pubertal processes. Saliva is less invasive than blood and advantageous for repeated measures, thus enabling researchers to collect several samples and derive a stable, basal hormone level. Measuring hormone levels in hair from the posterior vertex of the scalp is a more recent development in the puberty literature and has the advantage of being able to derive a cumulative basal hormone level that collapses across extraneous fluctuations. For example, whereas levels of hormones in saliva are affected by diurnal variation and reactivity to acute stressors (Ankarberg \& Norjavaara, 1999; Bao et al., 2003; Goodyer et al., 1998; Izawa et al., 2008), measuring hormones in hair has the advantage of observing presumably more stable, basal measures of hormone over a number of months (Wang et al., 2019). Measurement of androgens in hair is also reliable in samples of children and adolescents (Smith et al., 2018).

There are a number of ways to measure and organize pubertal processes from hormones and self-reported physical characteristics, however: 
1) how each method captures those underlying processes is not clear. For example, do self-reported and biological indicators of puberty better support a one-factor model, where there is one overall pubertal process, or a two-factor model, which is specific to the pubertal processes of adrenarche and gonadarche? And,

2) how much of the contribution to the variance in latent factors of puberty is due to underlying mechanistic processes compared to variance in the measurement type? For example, how correlated are the different measures of puberty with one another?

Understanding the structure of multi-method pubertal data may be useful for future research studies that attempt to understand both social and biological contributions of puberty to other outcomes such as psychopathology or brain development.

There is a paucity of studies comprehensively comparing measures of sex hormones (specifically, DHEA, testosterone, and in girls, estradiol) and physical characteristics of puberty (self- or clinician-reported). One landmark study (Shirtcliff et al., 2009) did so in boys and girls from ages 9-14 years (82 boys and 78 girls) and generally found that physical characteristics as measured by the Pubertal Development Scale (PDS; Petersen, Crockett, Richards, \& Boxer, 1988) were significantly correlated with basal levels of hormones (calculated using Empirical Bayes estimates) from 32 samples per participant, collected over 5 days. However, this correspondence varied across biological sex (physical exam did not correlate as well with salivary estradiol in girls), and further studies that focus on one sex, or stratify results by sex, may be informative. Furthermore, the authors conceptualized items and scores on the PDS as reflecting separable processes of adrenarche (pubic hair, body hair and skin changes items) and gonadarche (menarche, growth spurt, and breast development items). These scores have shown to be differentially associated with white matter microstructure in various brain regions (Herting et al., 2017); however, it is not yet clear if the items on the PDS and other measures of puberty fit best into this two-factor model, or if a model with one overarching factor of puberty is a better fit. In order to disentangle the contribution of multiple indicators of the dual processes of puberty, we need research on a sample in an appropriate age range that may show variability in both.

The current study explored multi-method cross-sectional pubertal data from a cohort of 174 early adolescent girls aged 10-13 years to develop a structural equation modelling framework of puberty for girls that included multiple measures of puberty and considered the separable pubertal processes of adrenarche and gonadarche. We considered adrenarcheal and gonadarcheal factors to capture the ongoing pubertal processes associated with adrenarche and gonadarche, i.e., originating in the adrenal gland and gonads, rather than the onset or timing of these developmental phases. We attempted to confirm a theory-driven approach by categorizing self-reported physical development (measured by the PDS and Morris \& Udry Line Drawings (LD); (Morris \& Udry, 1980)), and biological sex hormones (measured in saliva and hair) into expected adrenarcheal and gonadarcheal latent factors. Specifically, we expected PDS items 2 (underarm and pubic hair) and 3 (skin changes) and LD item 2 (pubic hair) to load onto an adrenarcheal factor and PDS items 1 (height growth), 4 (breast development), and 6 (onset of menstruation) and LD item 1 (breast development) to load onto a gonadracheal factor. We also predicted that salivary and hair DHEA and testosterone would load onto an adrenarcheal factor and salivary and hair estradiol would load onto a gonadarcheal factor. We explored model fits and loadings to compare this "two-factor model" separately for self-reported items and for biological sex hormones in both saliva and hair, as well as a two-factor model fit for all variables (self-report and hormones). For each model (self-report, hormones, and combined), we compared each of the two-factor models (adrenarcheal and gonadarcheal) to a one-factor model of general puberty to determine if the variables that are often used in developmental research can reliably differentiate between adrenarcheal and gonadarcheal processes. We also compared models that accounted for method variance by including covariances for method (PDS, LD, saliva, hair) to determine if items in each model were reflecting a pubertal process construct or if the variance was mainly due to the type of measure/questionnaire. Finally, we explored how strongly some measures of puberty, such as hair and saliva, or latent hormonal or self-report variables, were associated with one another, and how they were associated with chronological age, given that it is often used to represent maturity. Specifically, we examined how extracted latent estimates from self-reported measures of puberty were correlated with latent estimates from hormonal measures of puberty, and how much shared variance there was between age and latent pubertal factors. Our aim was to develop a framework of puberty for girls that includes multiple, best-fitting measures of puberty often used in developmental research 
while also accounting for how measurement type (self-report vs. hormones) and the processes of adrenarche and gonadarche fit into an overall model of puberty in girls. Overall, this exploratory study will assist researchers in deciding which measures to collect or how much weight to give each measure, depending on their research question.

Exploratory research question 1 (one- vs. two-factor models of puberty): When using multiple measures of pubertal stage (self-report, hormones) in a research study of early adolescent girls, do these measures reliably differentiate between adrenarcheal and gonadarcheal factors? For example, do the items on the PDS that theoretically assess processes of adrenarche and gonadarche fit well onto those latent factors, and/or do salivary and hair DHEA and testosterone load onto an adrenarcheal factor while estradiol loads onto a gonadarcheal factor? Or is a one-factor, general puberty model a better fit to the self-report and/or hormone data? In other words, how do the model fit indices and loadings change when we have one overall puberty factor compared to two adrenarcheal and gonadarcheal factors?

Exploratory research question 2 (method variance vs. pubertal process): How much is the variance in the latent factors (puberty or adrenarche and gonadarche) due to method (PDS, LD, saliva, hair) compared to underlying pubertal constructs that are not purely explained by the method of puberty measurement? In other words, how do the model fit indices and loadings change when we additionally account for covariance between items of the same method?

\section{Methods}

\section{Participants}

We enrolled 174 participants from the community for the ongoing longitudinal study Transitions in Adolescent Girls (TAG). Initially, 189 participants were recruited, but we excluded seven participants based on inclusion (fluent in English and report normal or corrected-to-normal vision) or exclusion criteria (had MRI contraindications; had been diagnosed with a developmental disability, psychotic and/or behavioral disorders including Autism Spectrum Disorders; reported taking psychotropic medications other than stimulants; or reported being or suspected they may be, pregnant), and another eight withdrew before completing the initial assessment. The current analysis includes data from the first wave of the study, collected from 2016-2018, when the girls were 10.0-13.0 years of age $\left(\mu_{\text {age }}=11.55\right.$ years, S.D. $\left.=0.81\right)$.

\section{Protocol}

We obtained written informed consent from the parent/guardian and written assent from the adolescent at the first of two laboratory visits comprising each wave of data collection. We trained the participants at this first visit to collect their saliva samples by providing them with a saliva kit and instructions to provide one saliva sample per week, which were then completed at home. At the second lab visit, the large majority of which were scheduled approximately one month after their first visit, participants returned the saliva kit, completed questionnaires on pubertal development, and a researcher collected a sample of their hair. Except for the saliva samples, all measures in these analyses (hair and questionnaires) were collected during the second session. The majority of participants completed four saliva samples; however, because some sessions were rescheduled, they completed up to seven samples. For this analysis, we used only the last four saliva samples given before the second session (days between first and last sample: $\mu=21.39$, S.D. $=6.13, \max =57$ ). We compensated participants for their time, and the Institutional Review Board at the University of Oregon approved all materials and procedures. For further details on the protocol for this ongoing longitudinal study, please refer to this document (https://psyarxiv.com/pvsbw/).

\section{Measures}

\section{Hormones.}

Saliva collection. To obtain a stable estimate of DHEA, testosterone $(T)$, and estradiol (E2), given that they cycle over days and weeks even prior to menarche (Zhang et al., 2008), we aimed to collect four saliva samples over the course of one month. Participants provided an average of four saliva samples at home (range: 1-7 samples). We instructed participants to collect the samples (2 $\mathrm{ml}$ each) via passive drool on 
Saturday mornings upon awakening and prior to the consumption of food or tooth brushing. They stored each vial in a cooler bag with Techni-ice in the family's freezer until they transported the cooler bag to the University of Oregon for the second lab visit, where we moved the samples to a $-80^{\circ} \mathrm{C}$ freezer in the lab until they were shipped (overnight with dry ice) to the Stress Physiology Investigative Team (SPIT) lab at lowa State University. Along with each saliva sample, participants completed a form that recorded the day and time of collection. They also noted if they were sick in the prior 24 hours, or had taken any medications during the previous day.

Hair collection. We collected approximately $100 \mathrm{mg}$ of hair near the posterior vertex of the scalp to be shipped at room temperature to the SPIT lab at lowa State University and then assayed for DHEA, testosterone, and estradiol using the methodology described by (Wang et al., 2019). We assayed the $5 \mathrm{~cm}$ closest to the scalp. Hair grows approximately $1 \mathrm{~cm}$ per month; therefore, the results are reflective of cumulative hormone levels from the prior 5 months. Participants also completed a brief survey about their hair, including whether they i) had curly hair, ii) had permed or colored their hair (along with relevant time frames), and iii) felt their hair was relatively sweaty ("Do you feel like you sweat relatively frequently/heavily on the head?"); however, these variables were not significantly associated with levels of hormones in our sample (Vijayakumar, et al., submitted, also available at https://psyarxiv.com/btf4y/), so we did not include these as covariates in the current analysis.

Self-reports of pubertal maturation. Participants completed the Morris \& Udry Line Drawings (LD) of self-reported Tanner Stage (Morris \& Udry, 1980). They viewed line drawings corresponding to the five Tanner stages of breast and pubic hair development, and were asked to choose images that best reflected their own development. Participants also completed the Pubertal Development Scale (PDS; (Petersen et al., 1988), which assesses height growth, body hair and skin changes, as well as breast development and menarche in females. It consists of five questions that are scored on a 4-point scale ranging from "no physical changes" to "development seems complete," (PDS items 1-4) with the only exception being the yes/no item on menarche (PDS item 6). There is an additional item on the PDS regarding social comparison (PDS item 5), as well as secondary answers that assess what the next closest response would be for each item, but we did not include these in the current analysis. All self-report items were specified as ordinal variables in the models.

\section{Processing and cleaning hormone data}

We assayed all hormones in hair and saliva in duplicate using ELISA kits (www.salimetrics.com). For hair, samples were washed, ground, extracted, reconstituted and assayed using methodology described by Wang et al. (2019); estradiol required a double-extraction to purify the sample prior to assay. We re-assayed saliva samples when the optical density intra-assay coefficient of variation (CV) was greater than $7 \%$, if enough sample was left over to do so. We assayed all hormones for each participant on the same day to minimize freeze-thaw cycles, and on the same plate, so as to minimize variation in hormone concentrations that may be attributable to plate differences (i.e., inter-assay CVs). All CVs reported are for the optical density wavelengths. The intra-assay CVs for saliva were $10.48 \%$ for DHEA, $1.80 \%$ for $\mathrm{T}$, and $7.76 \%$ for $\mathrm{E} 2$, and the intra-assay CVs for hair were $1.97 \%$ for DHEA, $2.70 \%$ for T, and $2.87 \%$ for E2. We processed the samples in two batches. For saliva, the inter-assay CVs for Batch 1 were $20.62 \%$ for DHEA, $10.23 \%$ for T, and $11.53 \%$ for E2, and for Batch 2 were $21.43 \%$ for DHEA, 8.34\% for T, and $15.55 \%$ for E2. For hair, the inter-assay CVs for Batch 1 were $2.42 \%$ for DHEA, $8.45 \%$ for T, and $7.81 \%$ for E2, and for Batch 2 were $17.08 \%$ for DHEA, $0.72 \%$ for $\mathrm{T}$, and there was only one plate for hair $\mathrm{E} 2$.

The number of mean salivary hormone concentrations (of the duplicates) that were non-detectable were as follows: DHEA: samples=38, participants=20; $\mathrm{T}$ : samples=3, participants=3; E2: samples=23, participants $=13$. We substituted salivary hormone concentrations that were non-detectable using rules listed in the Supplemental Material. We substituted hair hormone concentrations (mean of the duplicates) that were non-detectable and right-censored with the upper limit of the standards for that assay ( $\mathrm{N}$ : $\mathrm{DHEA}=0, \mathrm{~T}=2$, $\mathrm{E} 2=0$ ). No mean hair hormone concentrations were non-detectable and left-censored.

We also log-transformed hair and saliva hormone concentrations prior to analysis due to positive skew and kurtosis. Finally, we winsorized outlier values that were greater than 3 standard deviations from the mean (Hair N: DHEA=1; T=3, E2=1; Saliva N: DHEA=0, T=7, E2=7) to the value at $+3 \mathrm{SD}$ plus $0.01 \mathrm{pg} / \mathrm{ml}$ 
increments to maintain the ordinal value of the outliers. Code for cleaning the salivary hormone data is available at: https://github.com/dsnlab/TAG_scripts/tree/hormone cleaning/hormones .

\section{Statistical modelling}

We performed a series of confirmatory factor analyses (CFA) using the "lavaan" package (Rosseel, 2012) in $R$ version 3.5.0 (R Core Team, 2018) in three categories: 1) self-report models, 2) hormone models, and 3) full (i.e. self-report and hormone) models. Within each category, we tested if a two-factor (adrenal and gonadal; referred to as ADR and GON, respectively) or one-factor (pubertal; PUB) model provided better fit. We then compared the fit of models that did or did not include covariances between items within the same measure (i.e., PDS items ${ }^{1}$, LD items, saliva days, and hair). For model comparisons, we report the McDonald Fit Index (MFI; (McDonald, 1989), the comparative fit index (CFI), expected cross validation index (ECVI; (Browne \& Cudeck, 1989), standardized root mean squared residual (SRMR), and robust chi-squared tests $\left(\mathrm{X}^{2}\right)$. We considered smaller $\mathrm{X}^{2}$, SRMR, and ECVI values (closer to 0 ) and greater MFI and CFI values (closer to 1) to represent better fit. Refer to Figs. 1 - 7 in Results for visual theoretical representations of each model. Diagrams indicate standardized loadings. Code for the statistical modelling for this project is available at: https://github.com/dsnlab/TAG_puberty_W1/tree/2.0.0 (Release 2.0.0, DOI: 10.5281/zenodo.3696039).

For some models, we fitted data to post-hoc exploratory models, for example, to address low standardized loadings on some observed hair variables. We also tested associations between some latent variables in structural equation models, for example, between hair variables and latent salivary variables, as well as between hormone latent factors and self-report latent factors, in order to assess how much of the variance in one latent factor could be explained by another latent factor. By doing so we hoped to show how interchangeable each method of pubertal measurement might be in developmental research samples of early adolescent girls, while also addressing differences in each method where other measurement factors may be contributing additional variance. Finally, we tested how chronological age was associated with each latent factor, and compared this to traditional measures of composite scores of self-report data and averaging hormone data.

\section{Missingness.}

For all models that included ordinal data (self-report and full models), we used a pairwise maximum likelihood method (PML) to estimate parameters due to missing observations, to account for the ordinal nature of the PDS and LD items (and binary PDS item 6, menarche). For models with only continuous data (hormone models without any self-report indicators), we used maximum likelihood estimation (MLR) with robust (Huber-White) standard errors. For counts and percentages of missing data, please see Supplemental Material.

\footnotetext{
${ }^{1}$ We did not include covariances for PDS6, at binary item that asks if menarche has begun, as we considered this to be a different measure than the other PDS items, which ask if a process is not yet started, underway, or complete.
} 


\section{Results}

Each section includes results comparing one and two-factor models with and without covariances, first for the self-report measures, then for the hormone biospecimens, and then for all measures of puberty together. We also present several post-hoc exploratory analyses in each section, depending on the result of the a priori models, as explained above.

\section{Self-report models}

We first explored one-and two-factor models with and without covariances with the self-reported PDS and LD data, as shown in Figure 1.

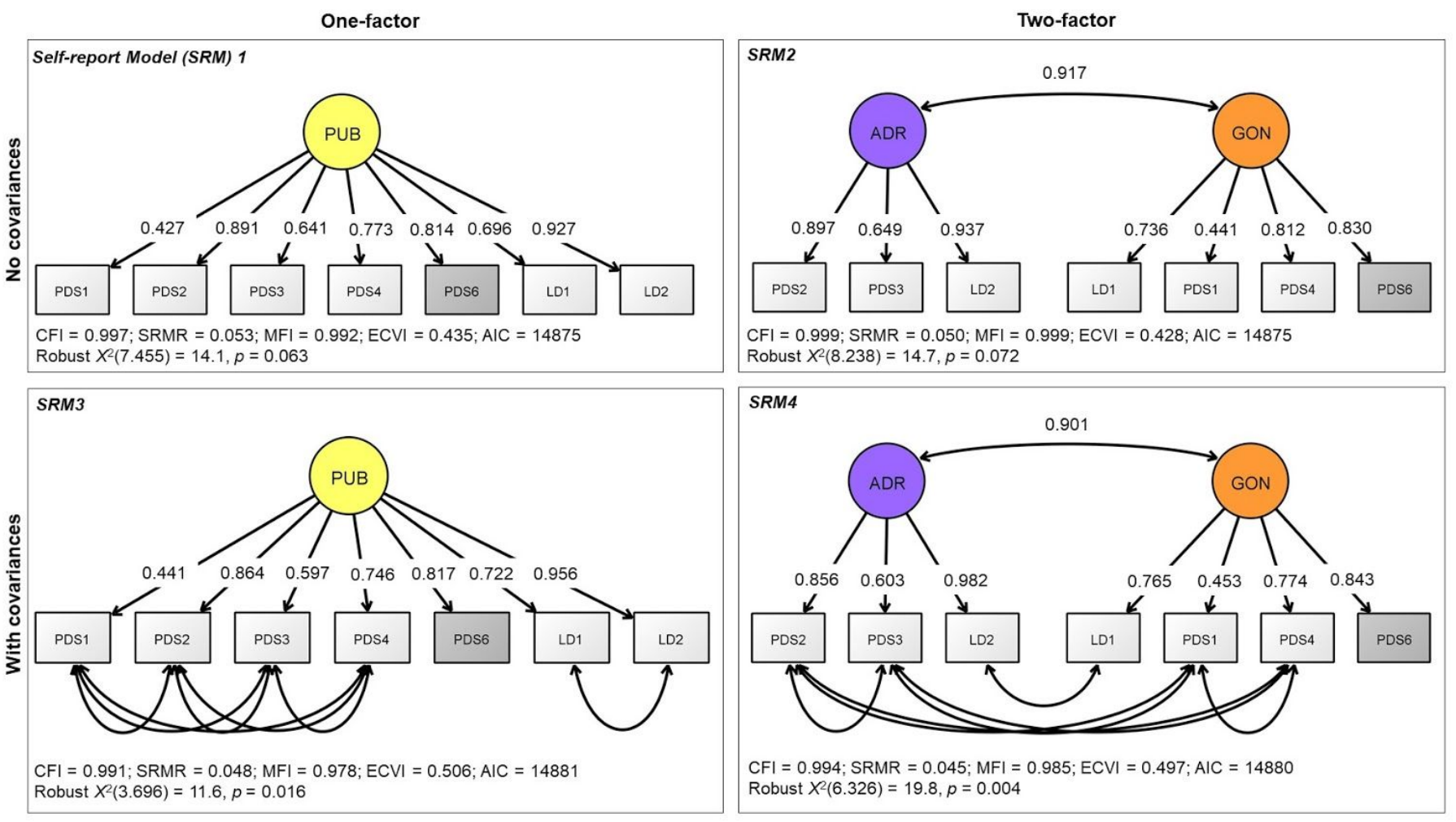

$P D S 1=$ Growth in height; $P D S 2=$ growth of body hair (underarm and pubic); $P D S 3=$ skin changes, especially pimples; PDS4 = breasts begun to grow; PDS6 = begun to menstruate $(Y / N)$; LD1 = breast development (line drawing); LD2 = pubic hair (line drawing). Self-report items are ordinal except for PDS6, which is binary.

Figure 1: One- and two-factor self-report models with and without covariances, including items from PDS and LD questionnaires.

Did a one- or two-factor model of puberty (without covariances) better fit the self-report data (SRM1 vs. SRM2)? There was a small but not statistically significant difference between SRM1 and SRM2, with SRM2 (two-factor) fitting marginally better $\left(X^{2}(1)=2.9, p=0.090\right)$. Model fit statistics were better for SRM2 for all indices except robust $X^{2}$. Loadings for all items but LD1 were also higher on the two-factor model. However, constraining the model to only one latent factor (PUB) did not fit the data worse. In other words, a more complex, two-factor model of puberty did not fit the self-report data better than the more parsimonious one-factor model.

For each of these models, did adding covariances (using correlated uniqueness models) between items from the same questionnaire change the model fit or item loadings, i.e., was the variance mostly accounted for by self-report measure (SRM1 vs. SRM3 and SRM2 vs. SRM4)? Using a correlated uniqueness model to account for additional variance due to questionnaire did not improve model fit for either the one- or two-factor model, and there was no significant difference between SRM1 and SRM3 or SRM2 and SRM4. All estimated covariances in SRM3 and SRM4 were not significantly different from zero (except for between ADR and GON, as expected); therefore, the models with and without covariances between method items are equivalent.

After accounting for measure covariances, did a one- or two-factor model of puberty better fit the self-report data (SRM3 vs. SRM4)? Similar to the models without covariances, there was a small but not statistically significant difference between SRM3 and 4, with SRM4 (two-factor) fitting marginally better $\left(X^{2}(1)=\right.$ 3.0, $p=0.082$ ). Fit indices except robust CFI and $X^{2}$ were slightly better. Loadings were higher except for PDS2. 


\section{Hormone models}

Next, we explored one-and two-factor models with and without covariances with the salivary and hair hormone data, as shown in Figure 2.


Figure 2: One- and two-factor hormone models with and without covariances, including all saliva and hair variables

Did a one- or two-factor model of puberty better fit the hormone data (HM1 vs. HM2)? We first fit models (not shown) that included each salivary hormone latent variable and observed hair hormone variable loading onto three latent hormone variables (DHEA, T, E2), before loading these onto one puberty factor or two ADR/GON factors. However, most of these models did not converge and were equivalent between the one- and two-factor models (because the latent GON variable is equivalent to a latent $E 2$ factor as there is only one hormone under GON). Therefore, we removed the second level latent hormone variables (DHEA, T, and E2) and loaded salivary DHEA, hair DHEA, salivary T, hair T, salivary E2, and hair E2 directly onto one PUB factor or two ADR and GON factors and compared those models, as shown. We also constrained loadings for hair DHEA to 0 , as these were initially negatively estimated. These models also did not fit well, and without covariances (HM1 vs. HM2), there was no significant difference between a one- and two-factor model $\left(X^{2}(1)=2.6, p=0.109\right)$. However, standardized loadings for both $E 2$ variables were higher with the two-factor model.

Did adding covariances change the model fit or item loadings, i.e., was the variance mostly accounted for by type of hormone measure (HM1 vs. HM3 and HM2 vs. HM4)? For both the one- and two-factor models, adding covariances between i) hair variables and ii) saliva variables collected on the same day, fitted the data significantly better (HM1 vs. HM3: $X^{2}(15)=166.9, p<0.001$; HM2 vs. HM4: $X^{2}(15)=167.1$, $p<0.001$ ). This suggests that covariances for biospecimen (i.e., hair and day of saliva collection) should be included.

After accounting for measure covariances, did a one- or two-factor model of puberty better fit the hormone data (HM3 vs. HM4)? Even after we included covariances for biospecimen, there was no significant difference between a one- and two-factor model $\left(\mathrm{X}^{2}(1)=2.2, p=0.134\right)$. However, again, standardized loadings for both E2 variables were higher with the two-factor model. Fit indices were also better for the two-factor model. 


\section{Additional post hoc explorations for hormone models:}

1) Removing hair DHEA and hair T (reduced hormone models; RHM). Given low standardized loadings for observed hair DHEA and hair T variables (consistently below 0.4 ) on the latent puberty factors, we tested additional post hoc models, with and without covariances, using only observed hair E2 variables and all saliva variables $(\mathrm{N}=171)$, as shown in Figure 3 . We also fit models with only saliva data and no hair data, given that hair E2 loadings were the lowest, and the results were similar (saliva-only models presented in Supplemental Materials).
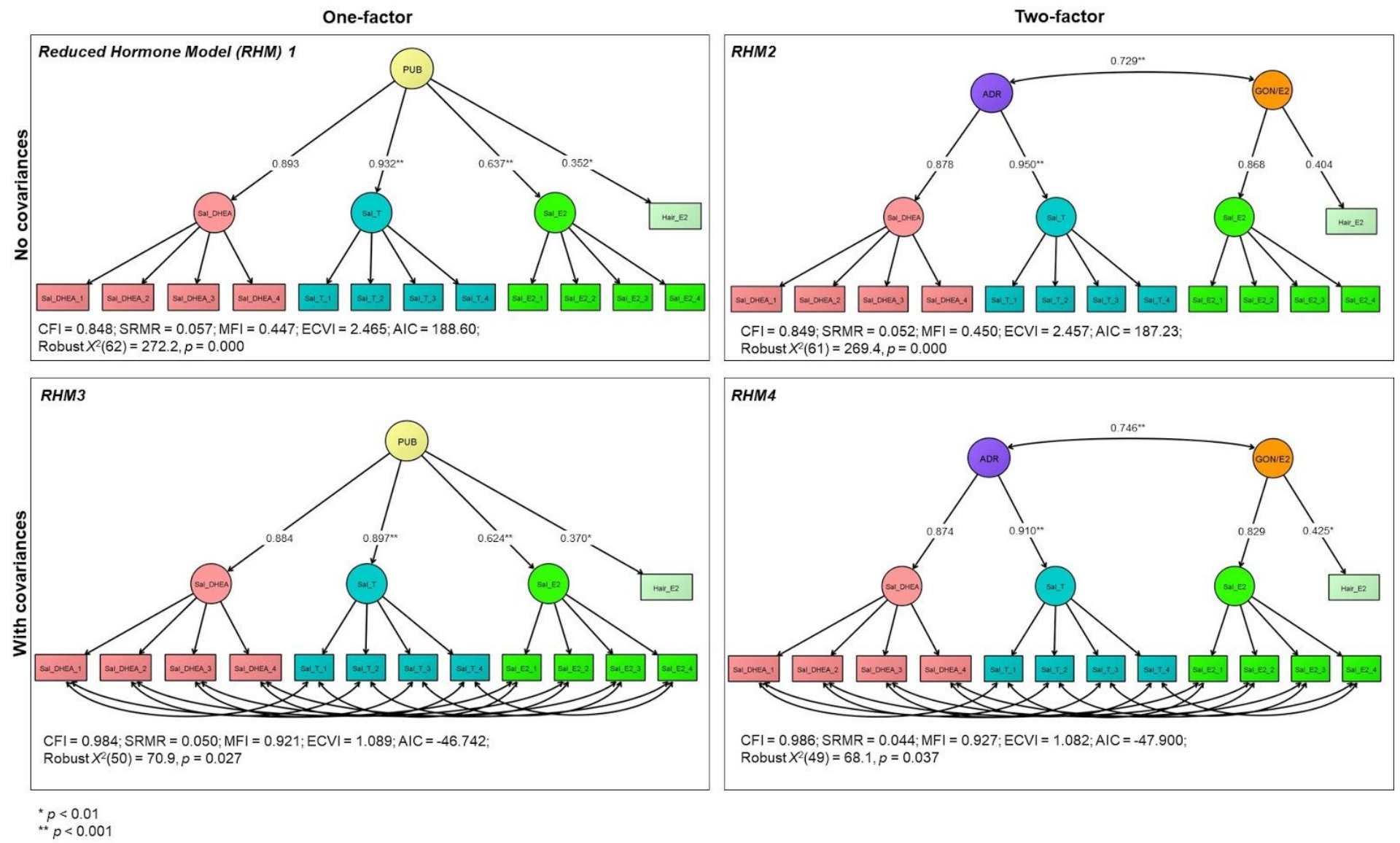

Figure 3: One- and two-factor reduced hormone models with and without covariances, including all salivary variables and only hair E2.

Did a one- or two-factor model of puberty better fit the reduced hormone data (RHM1 vs. RHM2)? Without covariances, there was no significant difference between a one- and two-factor model $\left(\mathrm{X}^{2}(1)\right.$ $=2.8, p=0.096)$. However, standardized loadings were higher and fit indices were better with the two-factor model.

Did adding covariances change the model fit or item loadings, i.e., was the variance mostly accounted for by type of hormone measure (RHM1 vs. RHM3 and RHM2 vs. RHM4)? Adding covariances between saliva variables collected on the same day fitted the data significantly better (RHM1 vs. RHM3: $X^{2}(12)$ $=142.9, p<0.001 ;$ RHM2 vs. RHM4: $\left.X^{2}(12)=143.5, p<0.001\right)$. This suggests that covariances for biospecimen should be included.

After accounting for measure covariances, did a one- or two-factor model of puberty better fit the reduced hormone data (RHM3 vs. RHM4)? Even after we included covariances for biospecimen, there was no significant difference between a one- and two-factor model $\left(X^{2}(1)=2.8, p=0.092\right)$. However, again, standardized loadings for all variables were higher and fit indices were better with the two-factor model. In other words, a more complex, two-factor model of puberty did not fit the hormone data better than the more parsimonious one-factor model.

2) Associations between hair and latent saliva factors (hair and saliva models; HSM). We also tested post hoc SEM hormone models to examine if observed levels of each hormone in hair were associated with the latent saliva factor for that hormone. Results are presented in Supplemental Material. 


\section{Full models}

Our full models (Figure 4) included all self-report and hormone variables, except hair DHEA and hair T, given the results of hormone models above. We tested one- and two-factor models. Based on results from self-report and hormone models, we did not use covariances for the self-report items, but we included covariances for biospecimen (saliva day). We also included second level latent variables for self-report (self-report ADR and self-report GON or self-report PUB). There was no significant difference between oneand two-factor models $\left(\mathrm{X}^{2}(1)=0.8, p=0.375\right)$. However, all fit indices for the one-factor model were better than for the two-factor model, contrary to the self-report only and hormone only models. Most importantly, a two-factor model was not a significantly better fit than the more parsimonious one-factor model of puberty. Using this more parsimonious one-factor model, the shared variance between the hormonal latent puberty factor and the self-reported latent puberty factor is $32 \%$ (i.e., $\left.(0.980 \times 0.576)^{\wedge} 2\right)$.
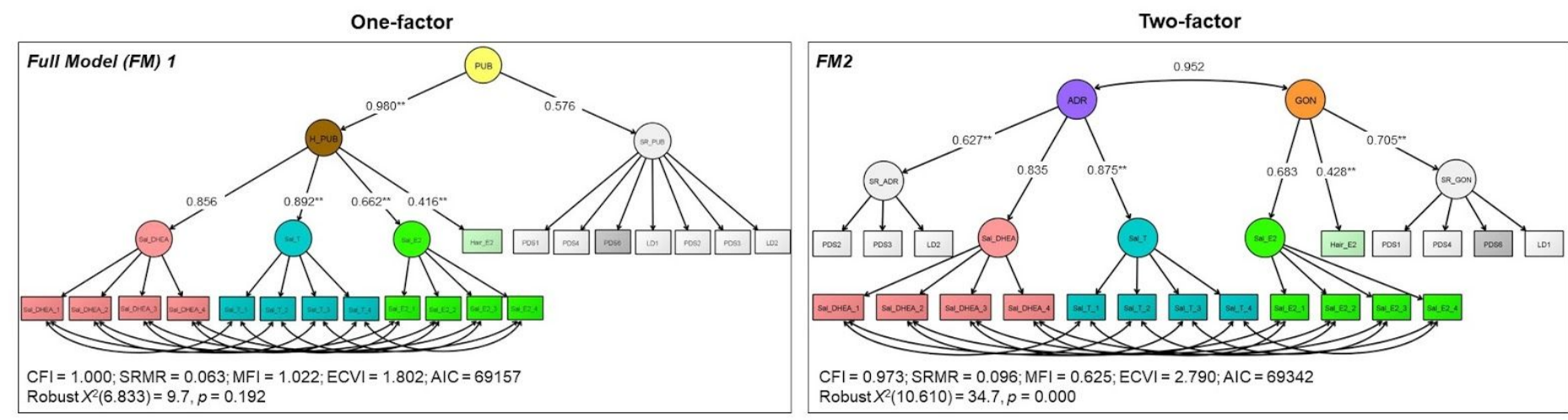

PDS1 = Growth in height; PDS2 = growth of body hair (underarm and pubic); $P D S 3=$ skin changes, especially pimples; PDS4 = breasts begun to grow; PDS6 = begun to menstruate (Y/N); LD1 = breast development (line drawing); LD2 = pubic hair (line drawing). Self-report items are ordinal except for PDS6, which is binary.

${ }^{*} p<0.01$

Figure 4: One- and two-factor full models combining salivary DHEA, T, E2, hair E2, and self-report items (PDS and LD)

Additional post hoc exploration of age for the two-factor full model. Given there was some variation in the chronological age of the sample (range 10.0-13.0 years, $\mu_{\text {age }}=11.55$ years, S.D. $=0.81$ ), and age is associated with pubertal development, we explored if age was a factor related to the ADR and GON latent factors that explained the positive correlations between those factors. First, we included age in the full model and allowed it to correlate with both the ADR and GON factors (Figure 5a), and found that it was significantly associated with ADR at $r=0.580(p<0.001,34 \%$ shared variance) and GON at $r=0.695(p<$ $0.001,48 \%$ shared variance) but that ADR and GON still correlated at $r=0.960(p<0.001,92 \%$ shared variance). Second, we fit a model that controlled for age, to assess if correlations were due to variation in pubertal timing, which is stage compared to same-age peers (Figure 5b). ADR and GON were still correlated at $r=0.951$ ( $p<0.001,90 \%$ shared variance). Third, we found that correlations between age and all latent one- and two-factor variables (from full and self-report only models) were uniformly higher than when using traditional scoring of questionnaires or mean values of hormones (results presented in Supplemental Material).

A

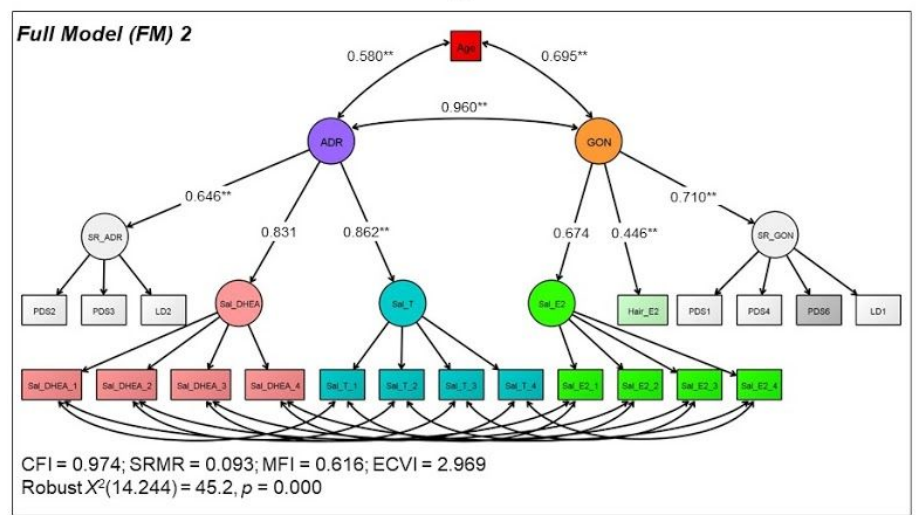

B

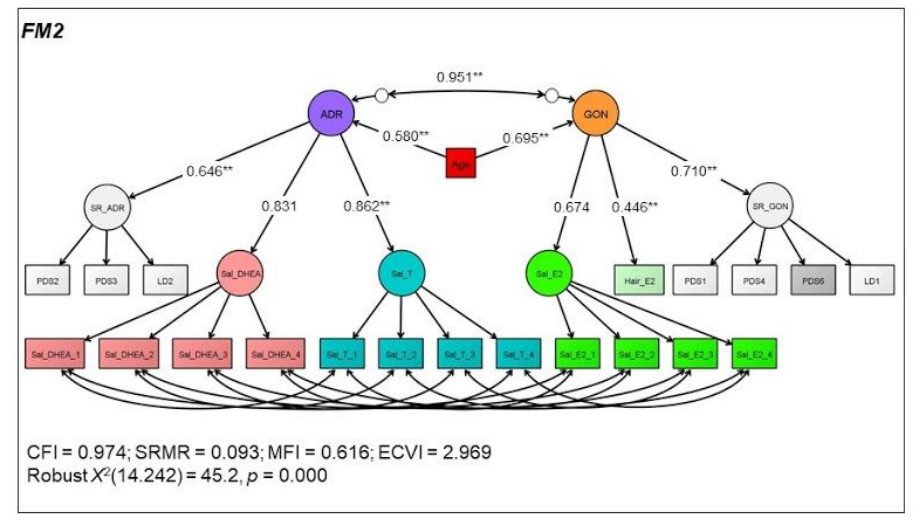

$P D S 1=$ Growth in height; $P D S 2$ = growth of body hair (underarm and pubic); $P D S 3=$ skin changes, especially pimples; PDS4 = breasts begun to grow; PDS6 = begun to menstruate (Y/N); $L D 1$ = breast development (line drawing); LD2 = pubic hair (line drawing). Self-report items are ordinal except for PDS6, which is binary.

${ }_{*}^{*} p<0.01$

** $p<0.001$

Figure 5: Two-factor full models combining salivary DHEA, T, E2, hair E2, and self-report items (PDS and LD), and a) correlating ADR, GON, and chronological age, and b) correlating ADR and GON both controlling for age 


\section{Discussion}

This exploratory study of multi-method pubertal variables collected from early adolescent girls suggests, overall, that self-reported pubertal characteristics and hormone levels from saliva and hair can estimate an overarching pubertal process as well as separate processes of adrenarche and gonadarche, with a few caveats.

\section{Self report data}

We found that a two-factor (ADR and GON) model was a slightly better fit to the data than one-factor (PUB), but that both models fit well and there was no appreciable difference between them; therefore, a more parsimonious, one-factor model of puberty was not a worse fit to the self-report data than a two-factor model. When we added covariances for questionnaire, model fit did not improve for either the one- or two-factor model, suggesting that latent factors likely capture variance due to underlying pubertal process constructs rather than questionnaire-specific method variance. This finding also suggests that items that are theorized to belong to separable pubertal processes can be used to study the biological processes of adrenarche and gonadarche in future puberty studies with girls of this age range, but can also be used to measure one pubertal process. We also found that the item about height growth (PDS1) had the lowest standardized loading. This is important for future studies, which could consider removing PDS1 from these constructs if adrenarche and gonadarche are specifically of interest, because this item may reflect a different process than adrenarche or gonadarche, and may not be synchronous for all girls (i.e., there may be individual differences in pubertal synchrony; for review, see (Mendle et al., 2019). On the other hand, it may contribute to a third process and the overall process of puberty, which may also be of interest to developmental science. Longitudinal studies would benefit from examining this item more carefully over time within individuals.

\section{Hormone data}

When we included all observed hair variables (DHEA, T, and E2), the models did not fit well, and there was no difference between the one and two-factor models. Including separate covariances for each day of saliva collection and for all observed hair variables significantly improved fit for the one- and two-factor models, suggesting that these account for some of the biospecimen variance. We found that loadings onto hormone latent factors were higher for saliva than for hair, but this may be because we had more salivary observed measures (four) than hair (one). Removing hair DHEA and hair T (thus only including observed saliva variables and hair E2) improved model fit indices. However, there was still no significant difference between the one- and two-factor models.

There are a number of possible reasons for the difference in loadings for hair E2 compared to the other hair variables. Researchers have successfully measured androgens in hair (Smith et al., 2018; Wang et al., 2019), yet protocols for measuring hormones in hair are less well-established than in saliva. Hair estradiol is an especially new avenue whose protocol requires a double-extraction to purify the sample prior to assaying as compared to a single-extraction for androgens. It is possible this additional extraction affects levels of hormones in the hair or their association with salivary estradiol. Furthermore, other noise in the hair hormone data (e.g., sweat, psychosocial stress) could affect androgens differently compared to estradiol, so future research could examine the differences in hormones across biospecimen more carefully, and should specifically examine whether or not the hair follicle acts as an endocrine organ for androgens during and after puberty (Randall et al., 2000; Zouboulis, 2009). Finally, the duration over which we measured saliva and hair in this sample differed. The four saliva samples were measured over approximately one month and combined into one latent factor. However, the single observed hair variable reflected levels of hormones that were aggregated over approximately five months of hair growth. It is possible that there were more fluctuations in the saliva (compared to hair) hormones for DHEA and T than for E2. In previous research with adults, more saliva samples were needed in order for hormone levels to significantly correspond with hair levels (Wang et al., 2019). Future research with adolescents should collect saliva and hair over similar time periods in order to more accurately compare levels of hormones between the two biospecimens for developmental research.

\section{Full models}


In models that included all variables (except hair DHEA and hair T, which did not load well in hormone-only models), a one-factor PUB model was slightly better fit than the two-factor ADR and GON model, but not statistically different. Therefore, depending on the research question, researchers are justified in loading multi-method biological and self-reported puberty data from girls aged $10-13$ years onto an overarching puberty factor, or separate adrenarche and gonadarche factors. However, the more parsimonious one-factor model was not a significantly worse fit than the two-factor model, and although not significant, the fit indices for the one-factor model were better. Future studies with larger sample sizes may be able to better fit two-factor models. The shared variance between the hormonal latent puberty factor and the self-reported latent puberty factor is $32 \%$. This suggests fairly good reliability between hormones and self-report puberty data, higher than previous studies that measured correspondence between salivary hormones and self-reported puberty (e.g., (Grotzinger et al., 2018; Shirtcliff et al., 2007, 2009), but also suggests that measuring puberty via hormones and via self-report questionnaires is not entirely redundant $68 \%$ not shared variance), and models of puberty require collecting both. This is also apparent in the two-factor model, where for the ADR factor especially, loadings for the self-report and saliva factors are not completely similar. Importantly, we suggest that collecting four saliva samples (one per week) in studies of adolescent development is feasible while still explaining enough variance between hormone and self-report latent factors.

Although our sample was fairly limited in the range of chronological age, there was some variation. In the two-factor full model, there was $34 \%$ and $48 \%$ shared variance between age and the ADR and GON factor, respectively. Therefore, although age was moderately associated with pubertal development, there was considerable additional variance not explained by age alone. Additionally, when controlling for age, ADR and GON were still strongly associated, with a $90 \%$ overlap in variance, suggesting that these factors are correlated when calculating them as pubertal timing (stage compared to same-age peers), as well as stage alone. Finally, we showed that correlations with age were higher when using the latent factors, whether for self-report questionnaire data alone, or for hormones, and correlations were highest when using all available self-report and biological measures (full results in Supplemental Table 2). Therefore, 1) full multi-method latent factor models are useful for measuring pubertal processes that are expected to correlate with age, but 2) even in the model where a latent pubertal factor correlated the most strongly with age, only $52 \%$ variance was shared between the pubertal factor and age, again suggesting that age is not a complete proxy for pubertal maturation. In other words, there may be no one "gold standard" measure for puberty, but rather pubertal development is comprised of multiple processes, and therefore, measured by multiple methods. Overall, by using multiple measures of pubertal development, this model captures pubertal processes that are somewhat distinct from age.

Further research should explore the impact of adding covariates to more comprehensively explore the factor structure of puberty, for example, other factors such as brain structure and function, acute stress or challenge (especially for salivary hormones), obesity, ethnicity, or self-perceptions of social expectations of physical characteristics compared to peers. It is also important to remember that including all measures representative of the pubertal processes (i.e, the multi-method process of puberty) may be important in order to estimate the "true score" representative of all pubertal processes, regardless of the correspondence between biospecimens and self-report questionnaires.

\section{Limitations}

Although this is the first comprehensive study of early adolescent girls that explores the structure of puberty from multiple methods of measuring hormones and self-reported physical characteristics, there are a few limitations to note for future research.

First, the current analysis is specific to our sample, which consisted of early adolescent girls aged 10 13 from Lane County in Oregon, U.S. As noted by other researchers, there are significant individual differences in pubertal processes, including differences dependent on race, ethnicity, sex, gender, and socioeconomic status (Ellis, 2004). Measurement of puberty in research currently suffers from a lack of inclusion of low-income youth, sexual, gender, racial, and ethnic minorities, and males (Deardorff et al., 2019; Mendle et al., 2019). A lack of diversity in hair and body type specifically limits the generalizability of our findings, even though our sample is representative for the local county. Also, results from samples measured at other ages during puberty (earlier at the start of adrenarche, or later when there is more variability in 
gonadal processes) may differ; we may find more differentiation of the two factors at different ages. We encourage future research to replicate our structural organization of multi-method puberty data with samples from other populations, especially those from large-scale, better-powered studies that may be more representative. This may include publicly available data from studies like the Adolescent Brain and Cognitive Development $(A B C D)$ study, although it should be noted that smaller-scale studies, like the current study, often have more comprehensive measures of puberty. For example, ABCD data includes both PDS and salivary hormones, but does not include line drawings of Tanner stages, hair hormones, or more than one saliva sample per wave. We need both types of studies to understand pubertal development.

Second, our analyses were cross-sectional because our aim was to organize indicators of pubertal stage during a snapshot of development. In addition, our cross-sectional data could have examined structure of indicators of pubertal timing (measures of pubertal stage controlling for age), but this was beyond the scope of a single paper. Instead, we simply showed that even when controlling for age (i.e., stage compared to same-age peers), correlations between adrenarcheal and gonadarcheal latent factors did not change substantially. Other developmental processes can be explored in longitudinal data within individuals, such as pubertal tempo (how quickly an individual completes a pubertal process from start to finish) and synchrony (correspondence between individual indicators of pubertal processes, i.e., the order in which indicators occur).

Third, there were a few possible confounders in our biological data that may have influenced our results. Saliva samples were collected by the participant at home, and although we instructed them to collect the sample first thing in the morning, some variation in adherence to time of day of collection could have influenced results. However, we included covariances for saliva samples collected on the same day to account for any confounders that may have been due to anything particular about that sample day, including variation in time of day. Additionally, as mentioned previously, we measured an aggregated level of hormone in hair over five months whereas saliva samples taken over a duration of one month were combined into a latent factor, and this difference in duration could have affected our results.

Finally, these analyses/models did not include covariates, such as body mass index and early life stress that tend to exhibit associations with pubertal development. Instead, this project tested models about the structure of pubertal development (including the full suite of factors that influence its measures in this sample), providing a foundation for future work to systematically investigate specific biopsychosocial factors related to puberty such as brain development, psychopathology, and other health outcomes. We additionally note that controlling for the full set of social, genetic, and environmental factors that influence pubertal development is neither possible with this data (because we lacked comprehensive measures), nor is it necessarily desirable. Instead, we conceive of puberty as a process comprised of social, genetic, and environmental components, and remaining variance after controlling for these factors may not be ecologically meaningful. The goal of the current exploratory study was not to look at individual differences in processes of puberty but rather to examine how multiple indices of puberty often used in developmental research covaried and loaded onto a priori theorized pubertal constructs. However, in addition to including covariates in models, future studies are justified to examine other outcomes associated with pubertal latent factors that could serve as validity evidence, such as additional physician-observed ratings (although it should be noted that often physician-observed ratings do not correlate well with all measures of puberty, such as estradiol in girls; Shirtcliff et al., 2009). Furthermore, there may be other factors correlated with pubertal latent factors, including sex, socioeconomic status, stressors, or perceived stress. The next step for puberty research is to use this structure to model individual differences in predictors and outcomes of puberty both cross-sectionally and with trajectories over time within subjects, in larger, better powered samples, examining not only pubertal stage, but timing and tempo, as well.

\section{Conclusions}

In this multi-method study of pubertal indicators in a sample of early adolescent girls, we found that self-reported puberty data from the PDS and line drawings, salivary hormones (DHEA, T, and E2), and hair E2 all load well onto theorized latent factors of pubertal processes. The item on height growth had the lowest standardized loading of the self-report items, so future research may need to reconsider what pubertal or developmental process that item is mapping onto. Considering we found differences in loadings for hair E2 compared to hair DHEA or T, future research should consider refining methodological protocol issues for hair 
measurement, such as single vs. double extraction. Overall, we did not find significant differences between models that included only one overarching pubertal factor and those that differentiated between adrenal and gonadal factors. Therefore, future research studies with early adolescent girls that have a theoretical reason to either combine or differentiate these processes are justified to combine multiple measures of puberty under these constructs, as long as the type of measurement matches the research question of interest.

\section{References}

Angold, A., Costello, E. J., \& Worthman, C. M. (1998). Puberty and depression: The roles of age, pubertal status and pubertal timing. Psychological Medicine, 28(1), 51-61.

Ankarberg, C., \& Norjavaara, E. (1999). Diurnal Rhythm of Testosterone Secretion before and throughout Puberty in Healthy Girls: Correlation with $17 \beta$-Estradiol and Dehydroepiandrosterone Sulfate ${ }^{1}$. The Journal of Clinical Endocrinology \& Metabolism, 84(3), 975-984.

https://doi.org/10.1210/jcem.84.3.5524

Bao, A.-M., Liu, R.-Y., van Someren, E. J. W., Hofman, M. A., Cao, Y.-X., \& Zhou, J.-N. (2003). Diurnal rhythm of free estradiol during the menstrual cycle. European Journal of Endocrinology, 148(2), 227-232.

Browne, M. W., \& Cudeck, R. (1989). Single Sample Cross-Validation Indices for Covariance Structures. Multivariate Behavioral Research, 24(4), 445-455. https://doi.org/10.1207/s15327906mbr2404_4

Byrne, M. L., Whittle, S., Vijayakumar, N., Dennison, M., Simmons, J. G., \& Allen, N. B. (2017). A systematic review of adrenarche as a sensitive period in neurobiological development and mental health.

Developmental Cognitive Neuroscience, 25. https://doi.org/10.1016/j.dcn.2016.12.004

Counts, D. R., Pescovitz, O. H., Barnes, K. M., Hench, K. D., Chrousos, G. P., Sherins, R. J., Comite, F., Loriaux, D. L., \& Cutler, G. B. (1987). Dissociation of Adrenarche and Gonadarche in Precocious Puberty and in Isolated Hypogonadotropic Hypogonadism. The Journal of Clinical Endocrinology \& Metabolism, 64(6), 1174-1178. https://doi.org/10.1210/jcem-64-6-1174

Deardorff, J., Hoyt, L. T., Carter, R., \& Shirtcliff, E. A. (2019). Next Steps in Puberty Research: Broadening the Lens Toward Understudied Populations. Journal of Research on Adolescence, 29(1), 133-154. https://doi.org/10.1111/jora.12402

Ellis, B. J. (2004). Timing of pubertal maturation in girls: An integrated life history approach. Psychological Bulletin, 130(6), 920-958. https://doi.org/10.1037/0033-2909.130.6.920

Goodyer, I. M., Herbert, J., \& Altham, P. M. (1998). Adrenal steroid secretion and major depression in 8- to 16-year-olds, III. Influence of cortisol/DHEA ratio at presentation on subsequent rates of disappointing life events and persistent major depression. Psychological Medicine, 28(2), 265-273.

Grotzinger, A. D., Mann, F. D., Patterson, M. W., Tackett, J. L., Tucker-Drob, E. M., \& Harden, K. P. (2018).

Hair and Salivary Testosterone, Hair Cortisol, and Externalizing Behaviors in Adolescents.

Psychological Science, 29(5), 688-699. https://doi.org/10.1177/0956797617742981

Hackney, A. C., \& Lane, A. R. (2015). Exercise and the Regulation of Endocrine Hormones. In Progress in Molecular Biology and Translational Science (Vol. 135, pp. 293-311). Elsevier.

https://doi.org/10.1016/bs.pmbts.2015.07.001

Herting, M. M., Kim, R., Uban, K. A., Kan, E., Binley, A., \& Sowell, E. R. (2017). Longitudinal changes in pubertal maturation and white matter microstructure. Psychoneuroendocrinology, 81, 70-79.

https://doi.org/10.1016/j.psyneuen.2017.03.017 
Izawa, S., Sugaya, N., Shirotsuki, K., Yamada, K. C., Ogawa, N., Ouchi, Y., Nagano, Y., Suzuki, K., \& Nomura, S. (2008). Salivary dehydroepiandrosterone secretion in response to acute psychosocial stress and its correlations with biological and psychological changes. Biological Psychology, 79(3), 294-298. https://doi.org/10.1016/j.biopsycho.2008.07.003

Ladouceur, C. D., Peper, J. S., Crone, E. A., \& Dahl, R. E. (2012). White matter development in adolescence: The influence of puberty and implications for affective disorders. Developmental Cognitive Neuroscience, 2(1), 36-54. https://doi.org/10.1016/j.dcn.2011.06.002

Lennartsson, A.-K., Kushnir, M. M., Bergquist, J., \& Jonsdottir, I. H. (2012). DHEA and DHEA-S response to acute psychosocial stress in healthy men and women. Biological Psychology, 90(2), 143-149. https://doi.org/10.1016/j.biopsycho.2012.03.003

McDonald, R. P. (1989). An index of goodness-of-fit based on noncentrality. Journal of Classification, 6(1), 97-103. https://doi.org/10.1007/BF01908590

Mendle, J., Beltz, A. M., Carter, R., \& Dorn, L. D. (2019). Understanding Puberty and Its Measurement: Ideas for Research in a New Generation. Journal of Research on Adolescence, 29(1), 82-95. https://doi.org/10.1111/jora.12371

Morris, N. M., \& Udry, J. R. (1980). Validation of a self-administered instrument to assess stage of adolescent development. Journal of Youth and Adolescence, 9(3), 271-280.

Oldehinkel, A. J., Verhulst, F. C., \& Ormel, J. (2011). Mental health problems during puberty: Tanner stage-related differences in specific symptoms. The TRAILS study. Journal of Adolescence, 34(1), 73-85. https://doi.org/10.1016/j.adolescence.2010.01.010

Paus, T., Keshavan, M., \& Giedd, J. N. (2008). Why do many psychiatric disorders emerge during adolescence? Nature Reviews Neuroscience, 9(12), 947-957. https://doi.org/10.1038/nrn2513

Petersen, A. C., Crockett, L., Richards, M., \& Boxer, A. (1988). A self-report measure of pubertal status: Reliability, validity, and initial norms. Journal of Youth and Adolescence, 17(2), 117-133.

R Core Team. (2018). R: A language and environment for statistical computing. R Foundation for Statistical Computing. https://www.r-project.org/

Randall, V. A., Hibberts, N. A., Thornton, M. J., Hamada, K., Merrick, A. E., Kato, S., Jenner, T. J., De Oliveira, I., \& Messenger, A. G. (2000). The Hair Follicle: A Paradoxical Androgen Target Organ. Hormone Research in Paediatrics, 54(5-6), 243-250. https://doi.org/10.1159/000053266

Rosseel, Y. (2012). lavaan: An R Package for Structural Equation Modeling. Journal of Statistical Software, 48(2). https://doi.org/10.18637/jss.v048.i02

Shirtcliff, E. A., Dahl, R. E., \& Pollak, S. D. (2009). Pubertal development: Correspondence between hormonal and physical development. Child Development, 80(2), 327-337. https://doi.org/10.1111/j.1467-8624.2009.01263.x

Shirtcliff, E. A., Zahn-Waxler, C., Klimes-Dougan, B., \& Slattery, M. (2007). Salivary dehydroepiandrosterone responsiveness to social challenge in adolescents with internalizing problems. Journal of Child Psychology and Psychiatry and Allied Disciplines, 48(6), 580-591. https://doi.org/10.1111/j.1469-7610.2006.01723.x

Smith, J. D., Johnson, K. A., Whittle, S., Allen, N. B., \& Simmons, J. G. (2018). Measurement of cortisol, dehydroepiandrosterone, and testosterone in the hair of children: Preliminary results and promising 
indications. Developmental Psychobiology. https://doi.org/10.1002/dev.21807

Soliman, A., Sanctis, V., \& Elalaily, R. (2014). Nutrition and pubertal development. Indian Journal of

Endocrinology and Metabolism, 18(7), 39. https://doi.org/10.4103/2230-8210.145073

Wan, W. (2012). Pubertal pathways and the relationship to anthropometric changes in childhood: The Fels longitudinal study. Open Journal of Pediatrics, 02(02), 118-126.

https://doi.org/10.4236/ojped.2012.22020

Wang, W., Moody, S. N., Kiesner, J., Tonon Appiani, A., Robertson, O. C., \& Shirtcliff, E. A. (2019). Assay validation of hair androgens across the menstrual cycle. Psychoneuroendocrinology, 101, 175-181. https://doi.org/10.1016/j.psyneuen.2018.10.029

Wohlwill, J. F., \& Palermo, D. S. (2014). The Study of Behavioral Development. Elsevier Science. http://qut.eblib.com.au/patron/FullRecord.aspx?p=1837440

Zhang, K., Pollack, S., Ghods, A., Dicken, C., Isaac, B., Adel, G., Zeitlian, G., \& Santoro, N. (2008). Onset of Ovulation after Menarche in Girls: A Longitudinal Study. The Journal of Clinical Endocrinology \& Metabolism, 93(4), 1186-1194. https://doi.org/10.1210/jc.2007-1846

Zouboulis, C. C. (2009). The skin as an endocrine organ. Dermato-Endocrinology, 1(5), 250-252.

https://doi.org/10.4161/derm.1.5.9499

\section{Supplemental Material}

\section{Non-detectable salivary hormone concentrations}

We substituted salivary hormone concentrations that were non-detectable and too low (i.e., left-censored; DHEA samples $=32$, T samples $=3$, E2 samples $=23$ ) using the following rules:

1) If other samples from the participant (i.e. from other sampling days) were also not detectable, the mean of all samples were replaced with the lower limit of sensitivity (DHEA: $5 \mathrm{pg} / \mathrm{ml}$; T: $1 \mathrm{pg} / \mathrm{ml}$; E2: 0.1 $\mathrm{pg} / \mathrm{ml})$;

2) If other samples from the participant were detectable and $50 \%$ or more of the remaining samples were below the lower interval of the inter-quartile range (IQR) for the distribution, the mean of the non-detectable sample was replaced with the lower limit of sensitivity for that assay;

3) If other samples from the participant were detectable and less than $50 \%$ of the remaining samples were below the IQR for the distribution, (i.e., most of the remaining samples were detectable and higher than the bottom quartile), the mean of the non-detectable sample was considered missing.

For mean salivary hormone concentrations that were too high (i.e., right-censored above the upper limit of the standards for that assay; DHEA only, samples =6), we applied the following rules:

1) If $50 \%$ or more of the remaining samples were above the upper IQR, the right censored sample was replaced with the upper limit of the standards for that assay (DHEA: $1000 \mathrm{pg} / \mathrm{ml}$; T: $600 \mathrm{pg} / \mathrm{ml}$; E2: 32 $\mathrm{pg} / \mathrm{ml})$.

2) If less than $50 \%$ of the remaining samples were above the upper IQR, the mean of the right censored sample was considered missing.

\section{Missing Data}

After following rules for replacement of hormone concentrations above, Supplemental Table 1 summarizes the counts and percentages of missing data. 


\begin{tabular}{|c|c|c|}
\hline Salivary DHEA sample 1 & 11 & 6.32 \\
\hline Salivary DHEA sample 2 & 11 & 6.32 \\
\hline Salivary DHEA sample 3 & 16 & 9.20 \\
\hline Salivary DHEA sample 4 & 26 & 14.94 \\
\hline Salivary T sample 1 & 8 & 4.60 \\
\hline Salivary T sample 2 & 9 & 5.17 \\
\hline Salivary T sample 3 & 15 & 8.62 \\
\hline Salivary T sample 4 & 26 & 14.94 \\
\hline Salivary E2 sample 1 & 9 & 5.172 \\
\hline Salivary E2 sample 2 & 9 & 5.172 \\
\hline Salivary E2 sample 3 & 14 & 8.046 \\
\hline Salivary E2 sample 4 & 25 & 14.37 \\
\hline Hair DHEA & 26 & 14.94 \\
\hline Hair T & 26 & 14.94 \\
\hline Hair E2 & 66 & 37.93 \\
\hline PDS1 (growth in height) & 11 & 6.32 \\
\hline PDS2 (growth of body hair) & 12 & 6.90 \\
\hline PDS3 (skin changes) & 9 & 5.17 \\
\hline PDS4 (breasts begun to grow) & 13 & 7.47 \\
\hline PDS6 (begun to menstruate) & 9 & 5.172414 \\
\hline LD1 (breast development) & 17 & 9.770115 \\
\hline LD2 (pubic hair) & 20 & 11.49425 \\
\hline
\end{tabular}

Supplemental Table 1: Rates of missingness for all variables

\section{Saliva-only models}

Given the low standardized loadings for hair E2, even in the Reduced Hormone Models, we also tested a one-factor model with and without covariances with saliva latent variables only. We only fit one-factor models because in a two-factor model, there would have been only one indicator on GON. Again, adding covariances between saliva variables collected on the same day fitted the data significantly better (SLM1 vs. SLM3: $X^{2}(12)$ $=143.0, \mathrm{p}<0.001)$. 

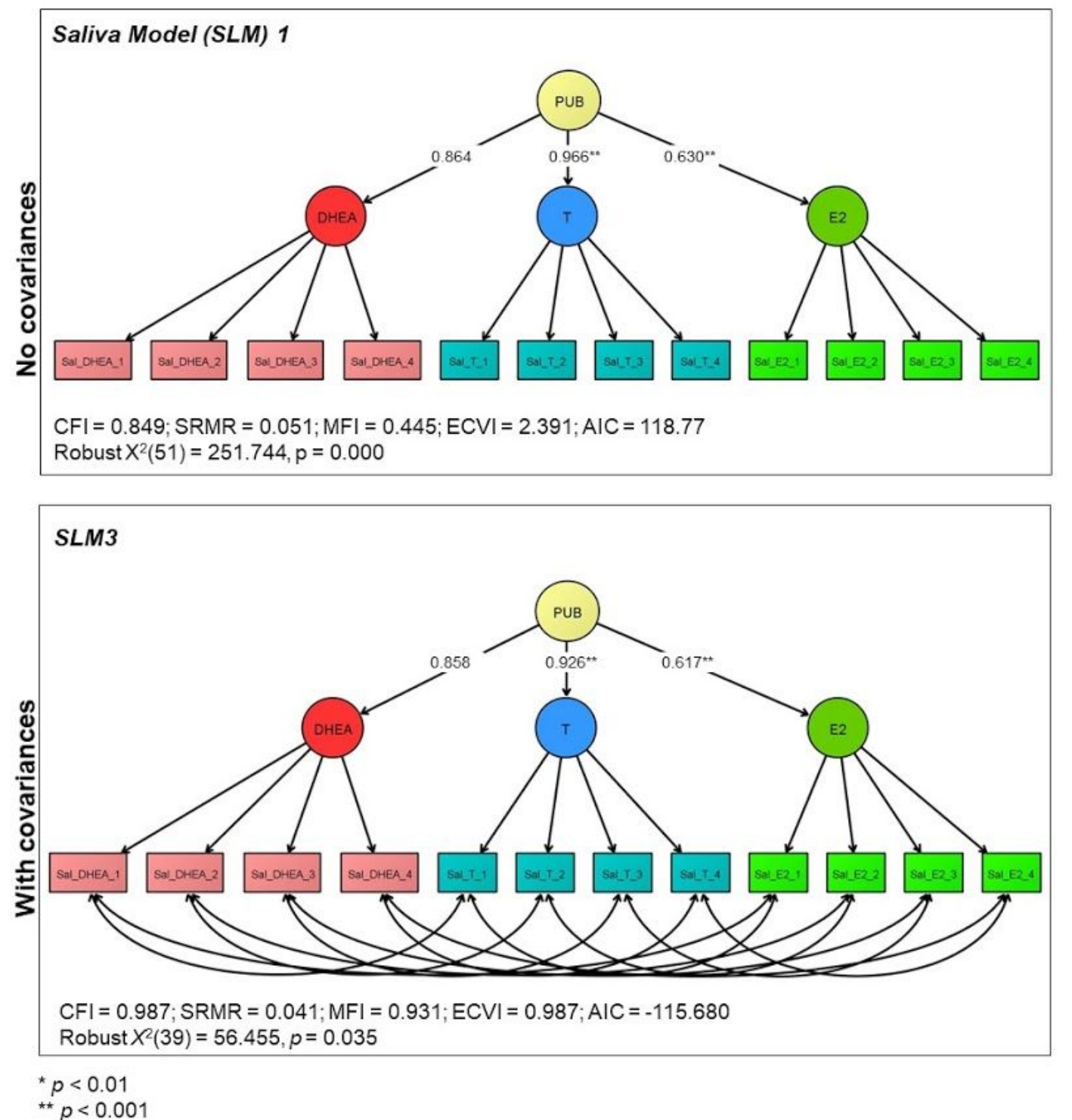

Supplemental Figure 1: One-factor saliva-only models.

\section{Associations between hair and latent saliva factors (hair and saliva models; HSM)}

We also tested post hoc SEM hormone models to examine if observed levels of each hormone in hair were associated with the latent saliva factor for that hormone. The first model (HSM1) did not include method factors for saliva and hair, and the second model (HSM2) included method factors. In both models, Hair E2 was significantly associated with the latent salivary E2 factor (HSM1: standardized estimate $=0.352, p=$ 0.002; HSM2: standardized estimate $=0.358, p=0.002$ ), but hair DHEA and T were not associated with their salivary counterparts. A pairwise likelihood ratio test comparing HSM1 to HSM2 revealed no significant difference in model fit: $X^{2}(0.89624)=1.0939, p=0.263$. Pairwise likelihood AIC and BIC indices were also similar across models (HSM1: PL AIC=19672 \& PL BIC=21260; HSM2: PL AIC=19668 \& PL BIC=21290). Overall, the observed hair measures were significantly associated with latent salivary factors (across four samples) for E2, but not for T or DHEA. Therefore, future studies may be justified to use hair E2 as a measure of puberty, but more research is needed to determine why $T$ and DHEA did not correspond with salivary counterparts as well, especially because this result is not consistent with previous research (Wang et al., 2019). 

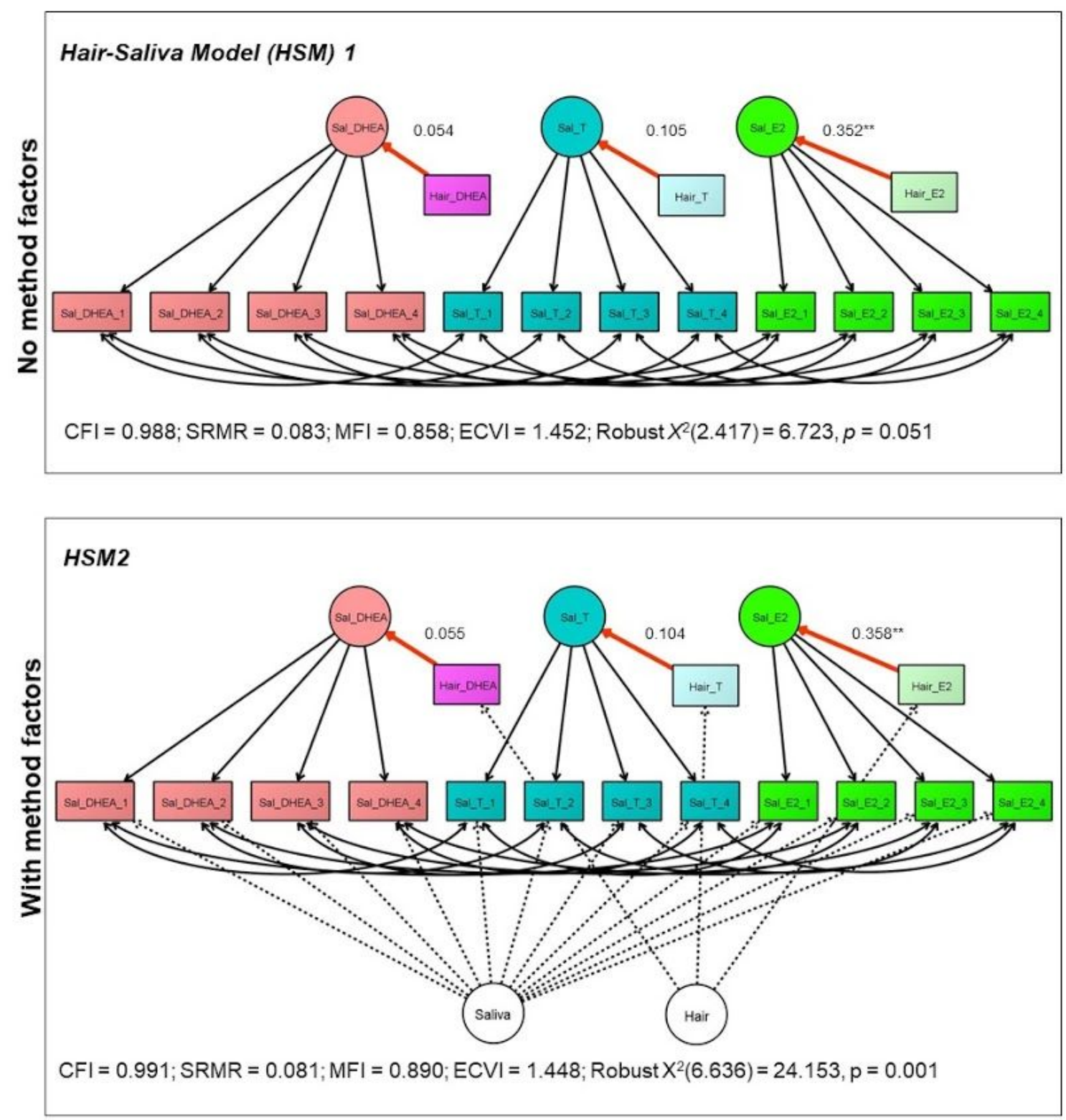

${ }^{\star \star} p<0.001$

Supplemental Figure 2: Structural equation models of observed hair variables predicting salivary latent factors, with and without method factors.

\section{Age correlations: comparing latent pubertal factors, traditional self-report scoring, and mean values of salivary hormones}

We calculated correlations between age and latent pubertal factors in the one- and two-factor full models (self-report, saliva, hair E2) and the self-report only models. We compared this to the composite scoring using the PDS and LD, which uses relevant theory-driven items on those measures (based on Shirtcliff, et al., 2009) to create an adrenarcheal (LD pubic hair and PDS adrenarcheal items) and a gonadarcheal (LD breast development and PDS gonadarcheal items) score, as well as an average overall pubertal stage score. We also compared age correlations with the latent factors to age correlations with the mean value of the four samples of relevant salivary hormones (DHEA, T, and E2 for the overall pubertal score, DHEA and T for the adrenarcheal score, and E2 only for the gonadarcheal score). Supplemental Table 2 shows that the associations with age when using latent factors were uniformly stronger than when using questionnaire scoring or mean salivary values, and latent factors from full models correlated with age more strongly than factors from the self-report models only. 


\begin{tabular}{|c|c|}
\hline Measure & $\mathbf{r}$ \\
\hline \multicolumn{2}{|l|}{ One-factor (PUB) } \\
\hline Pubertal composite (PDS \& LD) & 0.496 \\
\hline Latent PUB (self-report only: PDS \& LD) & 0.522 \\
\hline Mean salivary levels of pubertal hormones DHEA, T, E2 & 0.530 \\
\hline Latent PUB (full model: PDS, LD, hair E2, and salivary DHEA, T, and E2) & 0.724 \\
\hline \multicolumn{2}{|l|}{ Two-factor (ADR) } \\
\hline Adrenarcheal composite (PDS \& LD) & 0.494 \\
\hline Latent ADR (self-report only: PDS \& LD) & 0.527 \\
\hline Mean salivary levels of adrenarcheal hormones DHEA \& T & 0.497 \\
\hline Latent ADR (full model: PDS, LD, and salivary DHEA \& T) & 0.580 \\
\hline \multicolumn{2}{|l|}{ Two-factor (GON) } \\
\hline Gonadarcheal composite (PDS \& LD) & 0.406 \\
\hline Latent GON (self-report only: PDS \& LD) & 0.483 \\
\hline Mean salivary levels of gonadarcheal hormones E2 & 0.450 \\
\hline Latent GON (full model: PDS, LD, hair E2, and salivary E2) & 0.695 \\
\hline
\end{tabular}

Supplemental Table 2: Correlations with chronological age. All correlations were significant at $p<0.0001$ 